\title{
Microfluidic 2-D PAGE using multifunctional in situ polyacrylamide gels and discontinuous buffers $\dagger$
}

\author{
Shuang Yang, ${ }^{a c}$ Jikun Liu, ${ }^{a c}$ Cheng S. Lee ${ }^{b c}$ and Don L. DeVoe*ac \\ Received 2nd April 2008, Accepted 30th October 2008 \\ First published as an Advance Article on the web 19th November 2008 \\ DOI: $10.1039 / \mathrm{b805541f}$
}

\begin{abstract}
A two-dimensional microfluidic system is presented for intact protein separations combining isoelectric focusing (IEF) and sodium dodecyl sulfate (SDS) polyacrylamide gel electrophoresis (PAGE) employing in situ photopolymerized polyacrylamide (PAAm) gels. The PAAm gels are used for multiple functions. In addition to serving as a highly-resolving separation medium for gel electrophoresis, discrete polyacrylamide gel plugs are used to enable the efficient isolation of different on-chip media including anolyte, catholyte, and sample/ampholyte solutions for IEF. The gel plugs are demonstrated as on-chip reagent containers, holding defined quantities of SDS for on-chip SDSprotein complexation, and enabling the use of a discontinuous buffer system for sample band sharpening during SDS-PAGE. The 2-D chip also employs several unique design features including an angled isoelectric focusing channel to minimize sample tailing, and backbiasing channels designed to achieve uniform interdimensional sample transfer. Separation results using $E$. coli cell lysate are presented using a 10-channel chip with and without the discontinuous buffer system, with resolving power more than doubled in the former case. Further improvements in separation resolution are demonstrated using a 20-channel chip design.
\end{abstract}

\section{Introduction}

Despite the refinement of liquid chromatography and peptide mass fingerprinting techniques for protein analysis, 2-D PAGE separations of intact proteins remains a core technology for proteomic studies. In addition to enabling quantitative analysis of global protein levels, 2-D PAGE offers theoretical peak capacities around $10000,{ }^{1,2}$ with real-world detection of several thousand proteins commonly achieved in a single run. ${ }^{3}$ In 2-D PAGE, denatured intact proteins are separated on the basis of their charge state by isoelectric focusing (IEF), followed by a size-based separation using SDS-PAGE. While 2-D PAGE is most commonly practiced with backend analysis of proteins by mass spectrometry, 2-D PAGE expression maps alone can provide valuable insight for differential studies, including the analysis of post-translational modifications, by yielding information about the approximate isoelectric point (pI) and molecular weight (MW) of differentially expressed species within complex samples.

Overall, 2-D PAGE remains a labor intensive and low throughput process, with typical analysis times on the order of 1-2 days per run. ${ }^{2,4}$ This throughput limitation significantly constrains the utility of 2-D PAGE. In recent years, a number of efforts have targeted the development of microfluidic systems

\footnotetext{
${ }^{a}$ Department of Mechanical Engineering, University of Maryland, College Park, College Park, Maryland, 20742, USA

${ }^{b}$ Department of Chemistry and Biochemistry, University of Maryland, College Park, College Park, Maryland, 20742, USA

'Department of Bioengineering, University of Maryland, College Park, College Park, Maryland, 20742, USA. E-mail: ddev@umd.edu; Fax: +301-314-9477; Tel: +301-405-8125

$\uparrow$ Electronic supplementary information (ESI) available: Further details of SDS-PAGE separations. See DOI: 10.1039/b805541f
}

which mimic the functionality of 2-D PAGE in a miniaturized format, while promising significantly shorter analysis times and higher levels of automation. ${ }^{5-10}$ A common feature of these systems is the combination of a single microchannel for performing a first-dimension IEF separation, and an array of discrete second-dimension microchannels for capillary gel electrophoresis (CGE), with the second-dimension array replacing the traditional slab gel format for gel electrophoresis. Although the peak capacities of chip-based IEF/SDS-CGE systems remain lower than traditional 2-D PAGE, the application of microfluidics technology has successfully reduced separation times by 2 orders of magnitude, with substantially lower sample loading requirements than slab gels., ${ }^{9,10}$ Furthermore, recent advances including the demonstration of on-chip differential gel electrophoresis ${ }^{11}$ and separation repeatability comparable to slab gel analysis ${ }^{10}$ suggest that further developments may allow microfluidic 2-D separations to emerge as a viable high-throughput alternative to slab-gel 2-D PAGE.

To this end, a microfluidic IEF/SDS-PAGE separation system which employs a combination of multifunctional polyacrylamide (PAAm) gels and a discontinuous SDS-PAGE buffer system is described here. The PAAm gel is used as a highly-resolving separation medium for gel electrophoresis, while discrete PAAm gel plugs integrated into specific regions of the chip enable acid, base, and ampholyte solutions to be fully isolated prior to chip operation. The gel plugs also allow different separation buffers to be stored within the chip, allowing the use of a discontinuous buffer system chosen to enhance the resolving power of the second-dimension separation. The gel plugs are also employed as on-chip SDS containers, allowing defined volumes of SDS to be injected and complexed with the IEF-focused proteins without the need for external intervention. Integrating SDS directly into the chip obviates the need for manual injection as required in 
previous studies, ${ }^{6,10}$ improving automation of the system and providing photolithographic control over the amount of injected SDS.

The IEF channel itself possesses an angled geometry which has recently been shown to minimize sample tailing in multidimensional microfluidic systems. ${ }^{12}$ Additionally, the chip design employs optimized backbiasing channels which eliminate sample leakage and enable uniform sample transfer between the separation dimensions. This last feature is essential for eliminating injection current variations which have previously degraded the overall separation resolution and the uniformity of sample bands within the second dimension. Finally, improved SDS-PAGE separation resolution is achieved by taking advantage of sample stacking near the inlets of the second-dimension microchannels. Validation of the full 2-D system is presented using fluorescentlylabeled $E$. coli cell lysate, including a comparison between chip designs containing 10 and 20 parallel SDS-PAGE microchannels.

\section{Experimental section}

\section{Materials and chemicals}

Acrylamide (AAm), ammonium persulfate (APS), $N, N^{\prime}$-methylenebisacrylamide (Bis), $N, N, N^{\prime}, N^{\prime}$-tetramethylenediamine (TEMED), 3-(trimethoxysilyl)propyl methacrylate (TPM), poly(vinyl alcohol) (PVA, MW 130 000), 2-(cyclohexylamino)ethanesulfonic acid (CHES), glycine, fluorescein isothiocyanate (FITC), DL-dithiothreitol (DTT), iodoacetamide (IAM), triton X-100, and ampholyte ( $\mathrm{pH} \mathrm{3-10)}$ were purchased from Sigma-Aldrich Inc. (St. Louis, MO). $N, N$-Dimethylformamide (DMF) was purchased from EMD Chemicals Inc. (Gibbstown, NJ). Irgacure 2959 (I2959) was purchased from Ciba Corporation (Tarrytow, NY). Sodium dodecyl sulfate (SDS), urea, tris(hydroxymethyl)-aminomethane (Tris), methanol, isopropyl alcohol (IPA), concentrated sodium hydroxide, and concentrated hydrochloride acid $(\mathrm{HCl}, 36 \sim 38 \%)$ were purchased from Fisher Scientific (Pittsburgh, PA). High performance liquid chromatography grade DI water, purchased from Fisher Scientific, was used for sample and prepolymer solution preparation. The microfluidic chip substrate was fabricated from polymethylmethacrylate (PMMA) sheet (FF grade; Cyro, West Paterson, NJ), while the chip cover layer was fabricated using an ultraviolet-transmitting grade PMMA sheet (UVT grade; Spartech, Clayton, MO). Alexa-Fluor 488 model proteins, parvalbumin (MW $12 \mathrm{kDa}$ ), trypsin inhibitor (MW $21 \mathrm{kDa}$ ), ovalbumin (MW $45 \mathrm{kDa}$ ), and bovine serum albumin (BSA) (MW $66 \mathrm{kDa}$ ), were purchased from Invitrogen Corporation (Carlsbad, CA). Escherichia coli (E. coli) sample was purchased from Bio-Rad Laboratories (Hercules, CA).

\section{Microchip fabrication}

Channels and access reservoirs were directly machined into the chip substrate and cover layer, respectively, by a computer numerical control (CNC) milling machine (MDX-650A; Roland Corp., Lake Forest, CA). Acid and base reservoirs were first machined in the substrate layer using a $3 \mathrm{~mm}$ diameter end mill prior to channel fabrication. All channels were machined using a $125 \mu \mathrm{m}$ diameter end mill, with final channel geometries of
$150 \pm 3 \mu \mathrm{m}$ width by $140 \pm 5 \mu \mathrm{m}$ depth. The length of the IEF channel between the two outer backbiasing channels is $1.1 \mathrm{~cm}$, with each IEF channel segment $500 \mu \mathrm{m}$ long. The length of the CGE channels from the IEF channel to the detection region is $4 \mathrm{~cm}$. Access reservoirs were fabricated in the cover layer using a $1.8 \mathrm{~mm}$ diameter drill bit. Both PMMA plates were sequentially cleaned by methanol, IPA and DI water in a cleanroom environment prior to UV/ozone oxidization for $8 \mathrm{~min}$ using a commercial UV/ozone system (Novascan Technologies, Ames, IA). Following the process described by Zangmeister and Tarlov, ${ }^{13}$ the oxidized PMMA wafers were immediately immersed into a silanization solution consisting of $1.2 \mathrm{~mL}$ TPM and $0.2 \mathrm{~mL}$ $\mathrm{HCl}$ in $200 \mathrm{~mL}$ DI water, and sonicated within this solution for $1 \mathrm{~h}$. After nitrogen drying, the TPM-treated substrate and cover plates were thermally bonded using a hot press (Auto Four; Carver Inc., Wabash, IN) at $85^{\circ} \mathrm{C}$ under a pressure of $3.45 \mathrm{MPa}$ for $15 \mathrm{~min}$. The reservoirs of the resulting chips were sealed with blue semiconductor tape to avoid contamination prior to use.

\section{Gel fabrication}

A schematic image of the chip design is shown in Fig. 1. Crosslinked PAAm gels were fabricated within three different regions of the chip by sequential photopolymerization steps, using different acrylamide prepolymer solutions and individual photolithography masks to define each gel. Masking was performed using black tape applied to the chip surface prior to

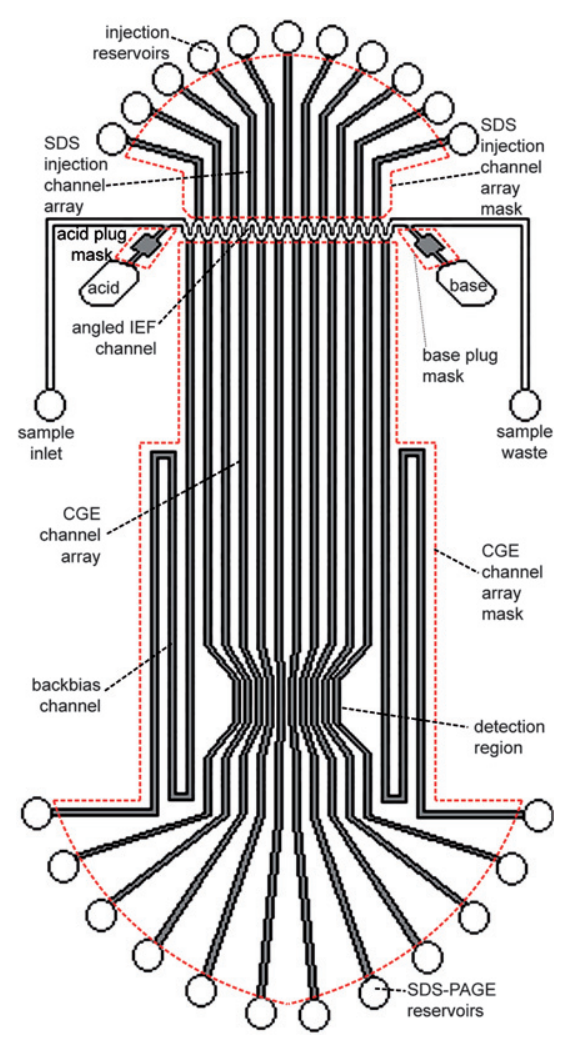

Fig. 1 Schematic of an IEF/SDS-PAGE separation chip combining PAAm sieving gel and gel plugs, an angled IEF channel design, and backbiasing channels. Photolithography masks used for patterning each of the gel regions are shown (dashed contours). 
Table 1 Prepolymer conditions for crosslinked PAAm gel formation, and buffer conditions in injection and SDS-PAGE reservoirs

\begin{tabular}{|c|c|c|c|c|c|}
\hline & Acid/base gel plugs & SDS-PAGE gel & SDS-PAGE reservoirs & Injection gel plugs & Injection reservoirs \\
\hline Bis $(w t \%)$ & 0.4 & 0.4 & - & 0.4 & - \\
\hline SDS $(w t \%)$ & - & 0.1 & 0.1 & 1.2 & 0.1 \\
\hline Buffer & $5 \mathrm{mM}$ Tris- $\mathrm{HCl}$ & $5 \mathrm{mM}$ Tris- $\mathrm{HCl}$ & 125 mM Tris- $\mathrm{HCl}$ & $\begin{array}{l}25 \mathrm{mM} \text { Tris }+192 \mathrm{mM} \\
\text { glycine }\end{array}$ & $\begin{array}{l}125 \mathrm{mM} \text { Tris }+\mathrm{HCl} 50 \mathrm{mM} \\
\text { glycine }\end{array}$ \\
\hline $\mathrm{pH}$ & 6.9 & 6.9 & 6.9 & $8.3^{8.8010}$ & 6.9 \\
\hline
\end{tabular}

UV exposure. A summary of conditions used for gel fabrication, including prepolymer composition, is provided in Table 1, together with details of the buffers used in the injection channel and SDS-PAGE reservoirs during second-dimension separations. Referring to Fig. 1, acid and base plug masks were used to pattern pressure-blocking gel plugs from a prepolymer solution consisting of AAm, Bis, and I2959 photoinitiator dissolved in $5 \mathrm{mM}$ Tris- $\mathrm{HCl}$ buffer. After photopolymerization with a total UV dose of $11 \mathrm{~J} / \mathrm{cm}^{2}$ delivered using a UV flood exposure instrument (PRX-1000; Tamarack Scientific Co., Corona, CA), unexposed prepolymer solution was removed by vacuum, and an acrylamide coating solution consisting of $6 \%$ AAm, 10\% APS, and 10\% TEMED in DI water at a volume ratio of $100: 1: 1$ was injected into the microchannel network. This coating solution was used to provide stress release at the gel/channel interface and prevent the formation of voids. After $15 \mathrm{~min}$, the coating solution was removed, followed by flushing with DI water. Next, the separation gel defined by the CGE channel array mask (Fig. 1) was fabricated using the same recipe as the acid and base gel plugs, but with SDS added to the buffer solution to yield a $0.1 \%$ background SDS concentration within the separation medium. After photopolymerization using a lower UV dose of $5.5 \mathrm{~J} / \mathrm{cm}^{2}$, the chip was again flushed with DI water to remove unreacted prepolymer. Finally, the gels defined by the SDS injection channel array mask were fabricated. The injection channel gels employed the same prepolymer recipe as the acid and base gel plugs, but using a different buffer with a higher concentration of SDS $(1.2 \%)$, allowing the gel plugs to serve as containers for defined quantities of SDS (see Table 1). These SDS packets are stored for later injection into the IEF channel for SDS-protein complexation following sample focusing. After the final UV exposure of the injection channel gels, the IEF channel was flushed and refilled with 4\% PVA for at least $30 \mathrm{~min}$ prior to sample introduction. The PVA coating served to minimize electroosmotic flow and protein adsorption during IEF. In situ gels for comparative tests using a continuous buffer system were prepared in $5 \mathrm{mM}$ Tris- $\mathrm{HCl}(\mathrm{pH}$ 6.9) for both the injection and SDS-PAGE gels.

\section{Sample preparation}

E. coli sample was prepared by dissolving $1 \mathrm{mg}$ FITC into $100 \mu \mathrm{L}$ DMF and mixing with reconstituted $E$. coli protein sample, which contained approximately $2.7 \mathrm{mg}$ of solid in $500 \mu \mathrm{L}$ DI water. The reaction was allowed to proceed in the dark overnight at room temperature. The total protein concentration of the fluorescein-labeled protein sample was determined to be $0.44 \mathrm{mg}$ / $\mathrm{mL}$ using a standard Bradford assay. ${ }^{14}$ To denature and reduce the E. coli sample, urea and DTT were thoroughly mixed with 1 $\mathrm{mL}$ of the sample solution in a vial with final concentrations of 8 $\mathrm{M}$ and $100 \mathrm{mM}$, respectively. The reaction was allowed to proceed in the dark overnight at room temperature. Subsequently, IAM was added to the solution to a concentration of $120 \mathrm{mM}$ to alkylate the denatured proteins, and the vial was placed in the dark for $1 \mathrm{~h}$ at room temperature. The resulting solution was diluted 20 times using a solution of urea, Triton $\mathrm{X}-100$, and ampholytes, for final concentrations of $8 \mathrm{M}, 10 \%$ $(\mathrm{w} / \mathrm{v})$, and $4 \%(\mathrm{v} / \mathrm{v})$ respectively.

Model proteins pre-labeled with Alexa-Fluor 488 were prepared in a similar manner. A total of $2 \mathrm{mg}$ protein sample (0.5 mg per protein) was dissolved into $400 \mu \mathrm{L}$ DI water, and 0.48 g urea and $15.4 \mathrm{mg}$ DTT were added into $100 \mu \mathrm{L}$ DI water. Both solutions were mixed and placed in a dark room overnight, followed by the addition of $0.277 \mathrm{~g}$ IAM. Finally, $100 \mu \mathrm{L}$ Triton $\mathrm{X}-100$ was added into the denatured sample. For SDS-PAGE separations alone, no ampholytes were employed, while for 2-D separations 4\% ampholytes were added. In each case, the concentrated proteins were diluted to $50 \mu \mathrm{g} / \mathrm{mL}$ by $8 \mathrm{M}$ urea and $10 \%$ triton $\mathrm{X}-100$ in DI water.

\section{IEF separations}

IEF was performed using platinum electrodes inserted into the acid and base reservoirs of the chip (Fig. 1) which were filled with $10 \mu \mathrm{L} 0.5 \mathrm{M}$ phosphoric acid and $10 \mu \mathrm{L} 0.5 \mathrm{M}$ sodium hydroxide, respectively. The electrodes were connected to the terminals of a high voltage power supply (CZE 1000R; Spellman, Plainview, $\mathrm{NY}$ ). A constant electric field of $250 \mathrm{~V} / \mathrm{cm}$ was employed to prevent protein precipitation during focusing. To minimize Joule heating, the initial current was limited to $15 \mu \mathrm{A}$, which decreased to less than $5 \mu \mathrm{A}$ within $2 \mathrm{~min}$. A focusing time of $3.5 \mathrm{~min}$ was used for all tests.

\section{SDS-PAGE separations}

Following IEF, two large common reservoirs formed from polymer were placed on top of the chip to connect all injection reservoirs together and all SDS-PAGE reservoirs together. A $2 \mathrm{~mL}$ volume of $125 \mathrm{mM}$ Tris- $\mathrm{HCl}$ buffer was pipetted into the common SDS-PAGE reservoir. For the continuous buffer system, the common injection reservoir was filled with $1 \mathrm{~mL}$ $125 \mathrm{mM}$ Tris- $\mathrm{HCl}$, while tests performed using the discontinuous buffer system employed $1 \mathrm{~mL} 125 \mathrm{mM}$ Tris- $\mathrm{HCl}+50 \mathrm{mM}$ 
glycine. SDS/PAGE was performed by applying a negative bias to the injection reservoir while holding the SDS-PAGE reservoir grounded using inserting platinum electrodes. A constant current of $5 \mu \mathrm{A}$ per channel was applied to chips containing 10 SDSPAGE channels, while a constant current of $10 \mu \mathrm{A}$ per channel was used for separations in the 20-channel chip.

Single dimension SDS-PAGE separations were performed using devices similar to the 2-D separation chips, but with only 5 SDS-PAGE channels and no acid/base reservoirs or connecting channels. In these tests, model proteins were injected into the IEF channel and transferred into the SDS-PAGE channels without first performing IEF separations. The length of the IEF channel segments between the centerlines of adjacent SDS-PAGE channels in the 5-channel chips was $300 \mu \mathrm{m}$, for a total sample loading of approximately $0.63 \mathrm{ng}$ per channel. The injection and SDS-PAGE reservoirs were filled in the same manner as the 2-D tests. A constant current of $5 \mu \mathrm{A}$ per channel was used for SDS-PAGE.

\section{Optical detection}

Optical detection was performed using an inverted fluorescence microscope (Nikon Eclipse TE2000s; Nikon Inc.; Melville, NY) with a $4 \times$ objective and a low-noise CCD camera (Coolsnap HQ2; Roper Scientific; Tucson, AZ) with a frame rate of $10 \mathrm{fps}$. The detection region shown in Fig. 1 is $40 \mathrm{~mm}$ away from IEF channel.

\section{Results and discussion}

\section{PAAm sieving gel}

While our previous efforts focused on using replaceable polyethylene oxide (PEO) as a sieving matrix in IEF/SDS-CGE chips, ${ }^{6,10}$ there are several disadvantages to this approach. At lower gel concentrations $(\sim 2 \%)$, PEO can mobilize due to small on-chip pressure gradients, resulting in unstable interfaces between the first and second dimension channels, but filling the CGE separation channel array at higher PEO concentrations $(>4 \%)$ is challenging due to the gel's high viscosity. Even when an ideal interface between the IEF and CGE separation media is initially achieved, inter-diffusion between PEO and sample/ ampholytes can rapidly deteriorate the interface, resulting in poor IEF resolution, sample loss, and irreproducible separation results. Furthermore, PEO separation performance is dependent on the operation temperature, ${ }^{15}$ with crystallization of the on-chip PEO solution typically observed following a single day of room-temperature storage. Both of these latter issues can further impact separation reproducibility.

Crosslinked PAAm fabricated by in situ photopolymerization is a sieving matrix which possesses a well-defined and tunable pore structure determined by the ratio of the monomer (AAm) and crosslinker (Bis) within the prepolymer solution. ${ }^{16}$ Originally explored for capillary separations, ${ }^{17,18}$ crosslinked PAAm has been widely used as a gel electrophoresis sieving medium in microfluidic platforms. ${ }^{\mathbf{8 , 9}, 19-22}$ The fabrication of long PAAm separation zones can result in voids along the gel-sidewall interface in silica capillaries due to shrinkage of the polymer chains during photopolymerization. ${ }^{23-25}$ To eliminate bubble formation, a layer of linear polyacrylamide (LPA) may be formed by bonding pedant methacrylate groups within the LPA to acrylic groups grown on the silica surface via TPM. The resulting LPA layer serves to relax interfacial shear forces produced due to gel shrinkage during polymerization, thereby preventing the formation of voids. Because native PMMA does not offer suitable functional groups for LPA attachment, UV-Ozone treatment was used here to oxidize the PMMA surface and generate hydroxide groups, which are further covalently bonded to methacryloxypropyltri-methoxysilane following the method of Zangmeister and Tarlov, enabling LPA to be anchored to the oxidized and MTP-treated PMMA surface. ${ }^{13,22,23}$ Even with this LPA surface coating, voids were consistently observed when using a reported PAAm recipe ${ }^{9,20}$ based on a prepolymer solution of $8 \%$ AAm, $0.03 \%$ Bis, and $0.24 \%$ I 2959 . However, by using LPA coating together with a modified gel recipe consisting of $4 \% \mathrm{AAm}, 0.4 \% \mathrm{Bis}$, and $0.12 \% \mathrm{I} 2959$, no voids were observed for gel plugs up to $8 \mathrm{~cm}$ long in the PMMA chips. Use of the optimized recipe without the LPA stress release layer resulted in voids for any PAAm gel longer than around $2 \mathrm{~cm}$.

\section{PAAm pseudo-valves}

In addition to the PAAm sieving medium, gel plugs fabricated adjacent to the acid/base reservoirs and within the injection channel array serve as pseudo-valves to prevent hydrodynamic flow of bulk solutions within the chip. Due to the small gel pore size, which is on the order of the protein molecules themselves, ${ }^{26,27}$ hydrodynamic flow through the PAAm gel pores is negligible. Although the PAAm is covalently linked to the channel sidewalls, bulk flow of solution can potentially occur by failure of the gel/wall interface, or structural failure of the highly crosslinked gel network. The pressure resistance of the gel plugs was characterized using a set of five single-channel chips with channels $110 \mu \mathrm{m}$ wide and $80 \mu \mathrm{m}$ deep containing a $300 \mu \mathrm{m}$ long PAAm gel plug. With one end of the channel connected to a syringe, a series of weights was applied to the syringe plunger until bulk flow was observed within the channel. Using this approach, an average burst pressure of $490 \mathrm{kPa}$ was determined. Considering the substantially longer gel plugs used in the IEF/ SDS-PAGE chip, even higher pressure resistance is expected. In addition, pressure resistance of the shorter pseudo-valves fabricated adjacent to the acid/base reservoirs (Fig. 1) was enhanced by increasing the local channel depth from $50 \mu \mathrm{m}$ to $140 \mu \mathrm{m}$, while also increasing the channel width from $150 \mu \mathrm{m}$ to $300 \mu \mathrm{m}$ to provide a mechanical constraint for these gel plugs. After injecting sample into the IEF channel and sealing the sample inlet and waste reservoirs, no bulk flow was observed even after 10 min equilibration, with stable and well-defined sample/gel interfaces maintained between the IEF channel and all injection and SDS-PAGE channels.

\section{SDS gel packets}

Another important use of the PAAm gel plugs is for reagent containers in the 2-D chip. In particular, the gel plugs formed in the injection microchannel array are photopolymerized with $1.2 \%$ SDS, which remains constrained within the gel until being mobilized by electrophoresis. Although diffusion of SDS out of 
the gel will occur over time, the period between chip preparation and testing $(\sim 1 \mathrm{~h})$ is sufficiently short to prevent this issue from affecting separation performance, and no intrusion of SDS into the separation channels during IEF was observed during testing. This approach allows well-defined and uniform quantities of SDS to be dispensed during the initial stage of SDS-PAGE, with SDS injected into the IEF channel complexing with focused proteins in real-time. Because complete binding of SDS with denatured proteins requires a $1.4: 1 \mathrm{SDS}$ : protein ratio, ${ }^{28}$ the total protein loading dictates the minimum amount of SDS which must be loaded into the gel packet. To avoid precipitation of proteins during IEF, sample concentration should be maintained below around $2 \mathrm{mg} / \mathrm{mL}$. In the present design, the IEF channel volume is $0.225 \mu \mathrm{L}$, thus a maximum sample loading of around $0.5 \mu \mathrm{g}$ is reasonable for the microfluidic chip, and a total SDS amount of $0.7 \mu \mathrm{g}$ is desired. Based on the SDS concentration and plug volume, the total amount of SDS within all eleven injection channels is approximately $28 \mu \mathrm{g}$, substantially more than the required $0.7 \mu \mathrm{g}$. The excess SDS ensures its high availability for protein binding, allowing the complexation process to occur in real-time without the need for incubation. ${ }^{\mathbf{1 0}}$

\section{IEF and sample transfer}

Integrated in situ PAAm gels have been previously used as pressure-blocking elements for 2-D separation chips, enabling sample to be loaded into the first-dimension microchannel with minimal intrusion into the second dimension channel array. ${ }^{8-10}$
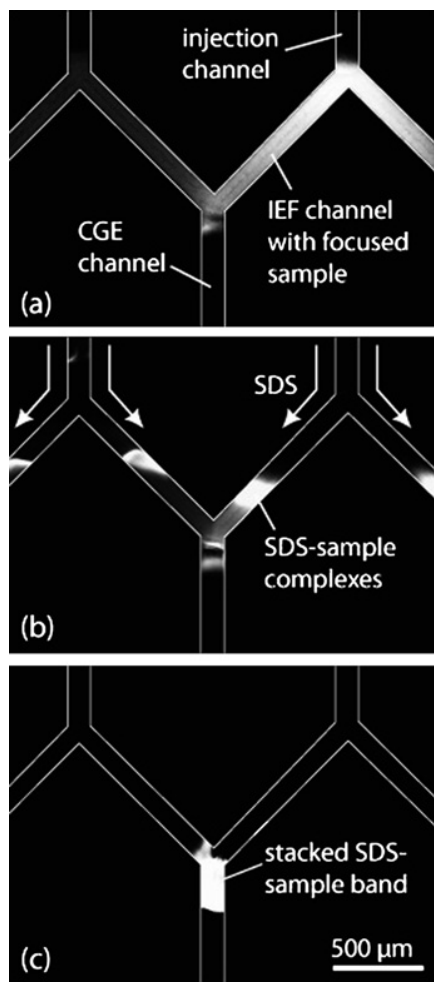

Fig. 2 Transfer of focused proteins showing (a) initial mobilization of SDS from the injection channels into the IEF channel, (b) real-time SDS-protein complexation, and (c) complete and uniform transfer of SDS-protein complexes to the SDS-PAGE dimension.
In particular, PAAm gel plugs have been shown to prevent hydrodynamic flow within the acid/base reservoirs, injection channels, and second-dimension separation channels, enabling highly-repeatable separations with less than 5\% variation in peak elution time for a chip containing five SDS-CGE microchannels. ${ }^{10}$ However, non-uniform injection of sample from the IEF channel to the SDS-CGE channel array remains problematic, resulting in significant broadening of the injected sample bands. Most critically, large electric field gradients across the width of a straight IEF channel can produce long sample tails during the injection process, preventing effective stacking following transfer to the second dimension and resulting in poor separation resolution. To provide a substantially more uniform electric field during sample transfer, each IEF channel segment was angled $45^{\circ}$ relative to its adjacent second dimension separation and injection channels (Fig. 2). This design can reduce electric field variations during sample transfer by a factor of 13 compared to a straight IEF channel. ${ }^{12}$

A detailed view of the sample transfer process following IEF is shown in Fig. 2. After the initial focusing step in Fig. 2(a), a negative bias is applied to the upper injection reservoirs with the lower SDS-PAGE reservoirs grounded, for an average electric field strength of $200 \mathrm{~V} / \mathrm{cm}$ during sample transfer. As SDS from the injection channel gel plugs enters the IEF channel, real-time complexation with the denatured proteins occurs, mobilizing the proteins towards the lower separation channels in Fig. 2(b). Even for complex samples, only minimal variations in migration speed of the SDS-protein complexes are typically observed among the different IEF channel segments. More importantly, as shown in Fig. 2(c), the entire sample is transferred into the SDS-PAGE channels, with no significant residual sample remaining within the IEF channel within $10 \mathrm{~s}$ of the initial SDS front reaching the proteins. This result compares favorably with previous 2-D separations performed using a straight IEF channel, where long sample tails up to several $\mathrm{mm}$ long were formed during sample transfer, severely degrading separation resolution. ${ }^{10}$

As shown in Fig. 1, the chip design also includes a long microchannel on either side of the second dimension array. These two channels, which are twice the length of the second dimension channels and filled with the same PAAm gel and buffer, are used to back-bias the IEF channel during sample transfer and SDS-PAGE separations. Back-biasing in the 2-D chip serves to eliminate injection non-uniformities, ensuring that sample bands enter the second dimension channel array simultaneously, while also preventing leakage of sample into the outermost separation channels within the array. ${ }^{\mathbf{1 2}}$

\section{SDS-PAGE separations}

In the commonly-used buffer system reported by Laemmli for slab gel SDS-PAGE, ${ }^{29}$ which extends the discontinuous buffer system originally developed by Ornstein ${ }^{30}$ and Davis, ${ }^{31}$ proteins are concentrated isotachophoretically within a large-pore stacking gel before being separated within an adjacent sieving gel. The Laemmli system employs chloride leading ions and glycinate trailing ions during the stacking process, with a transition from stacking to separation resulting from the use of a basic separation buffer several $\mathrm{pH}$ units higher than the stacking 
buffer. As the trailing glycinate ions enter the basic environment within the separation gel, their mobility increases substantially, allowing the ions to pass the slower proteins and finally resulting in a uniform separation buffer after stacking. In the microfluidic system, the multifunctional PAAm gels allow specific buffer conditions to be maintained within selected regions of the chip, such as the $\mathrm{pH}$ gradient required for the Laemmli buffer system. However, unlike slab gel SDS-PAGE, the 2-D chip does not readily allow for the integration of a large-pore stacking gel between the IEF channel and SDS-PAGE channel array. Furthermore, because of inhomogeneities in the pore size and distribution at the entrance to the photopolymerized PAAm sieving gel, substantial destacking can occur as sample bands mobilize into the sieving gel. Thus, to maximize separation efficiency in the 2-D chips, it is desirable to perform sample stacking after the SDS-protein complexes have entered the separation gel. To this end, a leading buffer containing chloride ions was placed within the PAAm separation gel, and a trailing buffer containing a low concentration of glycine was placed behind the SDS-complexed proteins during sample injection, with Tris as a common constituent of both solutions which were buffered to pH 6.9 (Table 1). In addition, buffer solution containing a high concentration of glycine, together with the SDS required for the second dimension separation, was placed within the injection channel gel plugs at $\mathrm{pH} 8.3$ to ensure a high initial mobility for glycinate ions within the injection channels. Soon after the SDS-protein complexes enter the separation gel, the high mobility glycinate ions sweep through the sample region, generating a transient electric field gradient which sharpens the individual protein bands. After the leading glycinate ions have traversed the sample region, size-based separation by SDS-PAGE continues within the background buffer containing a lower concentration of glycine. For the buffer conditions given in Table 1, full sharpening of the protein bands was typically observed within 5-10 $\mathrm{mm}$ after entering the CGE channels.

The effect of band sharpening using the heterogeneous buffer system can be seen in the single-dimension SDS-PAGE electropherograms shown in Fig. 3. Each of the four model proteins

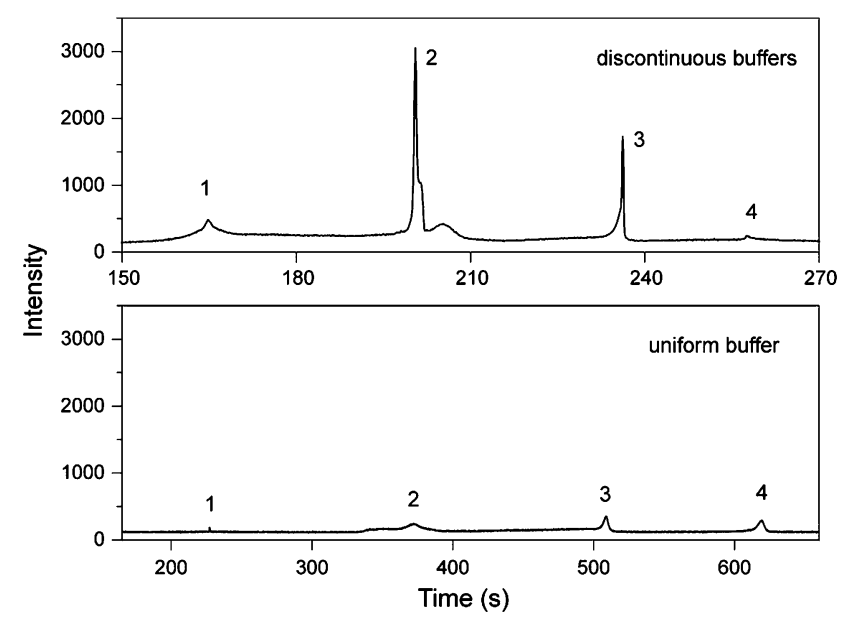

Fig. 3 On-chip SDS-PAGE of urea-denatured proteins in uniform and discontinuous buffer systems. The labeled electropherogram peaks correspond to (1) parvalbumin, $12 \mathrm{kDa}$; (2) trypsin inhibitor, $21 \mathrm{kDa}$; (3) ovalbumin, $45 \mathrm{kDa}$; (4) BSA, $66 \mathrm{kDa}$. Length to detector $=4 \mathrm{~cm}, \mathrm{E}=100 \mathrm{~V} / \mathrm{cm}$. separated in these experiments (parvalbumin, trypsin inhibitor, ovalbumin, and BSA) are resolved in both buffer systems with similar relative elution times, but with significantly higher signalto-noise ratios when using discontinuous buffers. The bands are also narrower, leading to a higher peak capacity for the discontinuous SDS-PAGE separation. Defining the width of a sample band as the time for the half-height full-width of a band to pass the detector, normalized to the total time between the first and last peaks of each electropherogram, the average peak capacity for the discontinuous buffer separation measured over five runs across all model proteins was 144, compared with an estimated peak capacity of only 27 for the continuous buffer case (see ESI $\uparrow$ for data used in these calculations). Note that due to the low signal-to-noise ratios in the case of the continuous buffer system, the actual increase in peak capacity is likely higher than the estimated $>5$ times improvement.

\section{IEF/SDS-PAGE separations}

Full 2-D separations of $E$. coli cell lysate were performed using both uniform and discontinuous buffer systems. Experiments were first performed using a chip containing 10 parallel SDS-CGE channels, with the results shown in Fig. 4((a), (b)). In each experiment, electropherograms extracted from the SDS-PAGE channel array were used to generate a pseudo-gel image similar to a conventional 2-D PAGE image revealing MW and $\mathrm{pI}$ for each protein peak, but with the MW axis replaced by elution time within the 2-D chip. Using a uniform buffer system consisting of $5 \mathrm{mM}$ Tris- $\mathrm{HCl}(\mathrm{pH}$ 6.9), approximately 40 relatively low-intensity peaks possessing a signal-to-noise ratio greater than 5 were detected, as shown in Fig. 4(a). In contrast, the use of discontinuous buffers provided significantly higher resolving power in the 10-channel chip, with a similar overall peak pattern but approximately twice the number of total peaks as shown in Fig. 4(b). Following background subtraction for both cases, the dynamic range for the chip employing the discontinuous buffer system is noticeably higher, with $18 \%$ higher average peak intensities.

Further improvements in resolving power were achieved by increasing the density of channels in the second-dimension SDS-PAGE array. Capillary or microchannel IEF is a highlyresolving electrokinetic separation technique, with maximum resolution dictated by precipitation as sample concentration increases during focusing. Typical peak capacities on the order of 60 (based on four model proteins IEF separation) were measured for the relatively short $(1.1 \mathrm{~cm})$ IEF channel used in the 2-D chip design. In order to take full advantage of IEF, it may be argued that the focused proteins should be sampled into the second dimension near the resolution limit of the IEF separation, suggesting that around 60 SDS-PAGE channels would provide optimal peak capacity for the overall separation. However, since detection sensitivity is a function of analyte concentration, it is advantageous to sample the focused proteins below the IEF peak capacity to prevent large numbers of focused sample bands from being split into multiple SDS-PAGE channels, thereby reducing maximum concentration within each channel at the downstream detector. With this constraint in mind, E. coli lysate separations were performed using a chip containing 20 SDS-PAGE channels, resulting in the pseudo-gel image shown in Fig. 4(c). The 


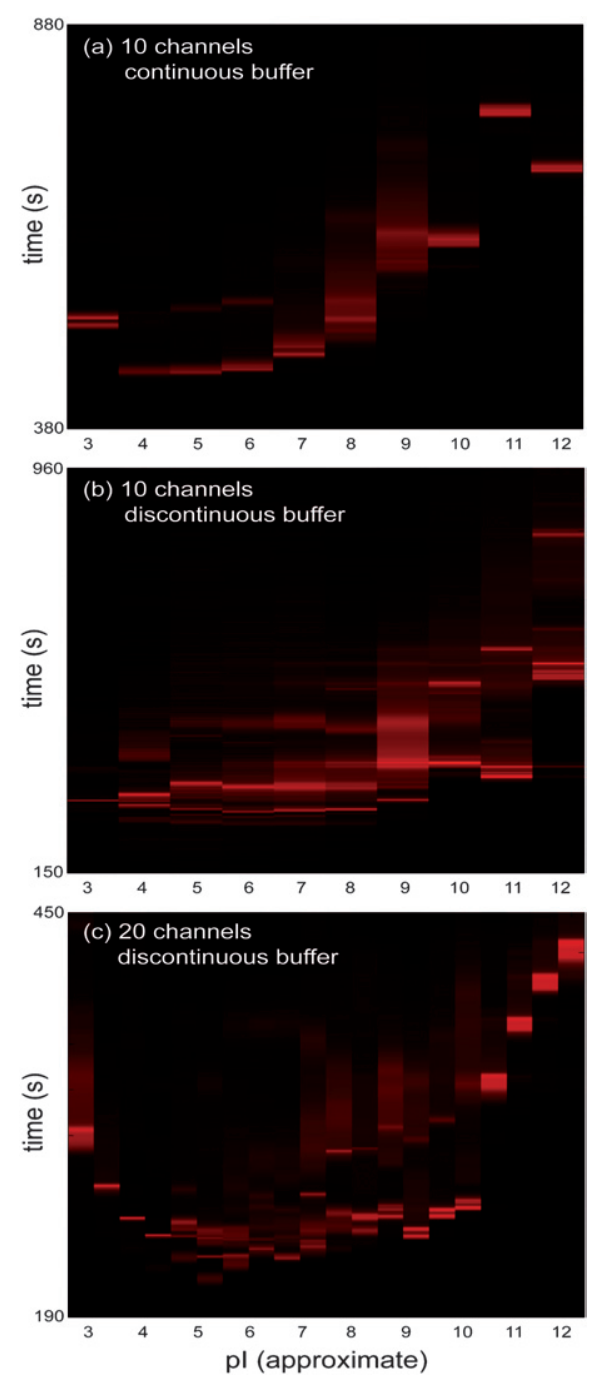

Fig. 4 Pseudo-gel images of IEF/SDS-PAGE E. coli cell lysate separations using (a) continuous buffers in a 10-channel SDS-PAGE chip, (b) discontinuous buffers in a 10-channel chip, and (c) discontinuous buffers in a 20-channel chip.

20-channel chip design was similar to the 10-channel chips, but with an IEF channel twice as long to accommodate the same inter-channel spacing. The electric field used for IEF was identical for both cases. The resolving power of an IEF separation $(\Delta p I)$ can be expressed as, ${ }^{32,33}$

$$
\Delta p I=3 \sqrt{\frac{D}{E} \frac{d p H / d x}{-d \mu / d p H}}
$$

where $D$ is the protein diffusion coefficient, $E$ is the electric field strength, $\mu$ is the electrophoretic mobility, $d p H / d x$ is the $\mathrm{pH}$ gradient along the channel, and $-d \mu / d p H$ is the mobility change along the $\mathrm{pH}$ gradient. Assuming $d \mu / d p H, D$, and $E$ remain constant, $\Delta p I$ decreases by $29 \%$ when channel length is doubled, leading to a moderate increase in peak capacity for the separation. However, because the sampling resolution is primarily defined by the density of SDS-PAGE channels, the improved resolving power resulting from the longer IEF channel is not expected to significantly affect the overall separation results.
Thus the same approximate $\mathrm{pI}$ range was sampled by the seconddimension microchannels, but with twice the sampling resolution for the 20-channel design.

While the same electric field was used for IEF, the 20-channel SDS-PAGE separation was performed using a constant current of $10 \mu \mathrm{A}$ per channel, twice that of the 10-channel design, leading to more rapid elution of proteins past the detection region. The resulting separation using the 20 -channel chip reveals a more complex pattern of peaks, with only a few bands which appear to co-elute across adjacent SDS-PAGE channels. It is notable that the 20-channel chip also appears to reveal features at the extreme ends of the $\mathrm{pH}$ range which were not apparent in the 10-channel design, suggesting that a wider $\mathrm{pH}$ range was sampled for the 20-channel case with a higher resolution in the shallow $\mathrm{pH}$ gradient. It is possible that the additional channel junctions present in the higher density chip led to deviations in the electric field that resulted in a wider $\mathrm{pH}$ gradient between the outer SDSPAGE channels.

Based on the average measured SDS-PAGE peak capacity of 144 using the discontinuous buffer system, and noting that the number of IEF channels is well below the theoretical peak capacity of the IEF separation, the 20-channel chip can provide an estimated 2-D peak capacity of 2880 (144 times 20). However, it should be noted that in several instances within the 20-channel chip, groups of sample bands within adjacent channels appear to exhibit similar patterns, but with different migration times. While such patterns are not unusual in traditional 2-D gels, it is also possible that these multiple bands reflect a degree of nonorthagonality between the on-chip separations.

\section{Separation reproducibility}

Since the various separation reagents and buffers were stored on-chip within the multifunctional gels, repeated experiments using a single chip could not be performed to evaluate run-to-run reproducibility, and separation reproducibilty across multiple chips was limited by several factors. For example, differences in channel dimensions commonly result from the direct machining process used for chip fabrication, leading to channel widths and depths with measured variations of $\pm 3 \mu \mathrm{m}$ and $\pm 5 \mu \mathrm{m}$, respectively. These dimensional errors result in channel-tochannel electrical resistance variations as large as 10\%, leading to irreproducible electric field distributions between chips.

An additional factor affecting reproducibility is variations in the quality of the polyacrylamide sieving gels. Although UV exposure conditions were identical for all chips used in this work, both intra-chip and inter-chip differences in gel properties can occur. A challenging requirement during chip fabrication is the reduction of bulk flow of prepolymer solutions prior to gel photopolymerization. A chip containing 20 SDS-PAGE channels requires a total of 46 interconnected on-chip reservoirs, and incomplete elimination of pressure gradients between these reservoirs can readily generate unwanted bulk flows during photopolymerization that affect gel interfaces at the entrances to the SDS-PAGE channels. Displaced and poorly defined interfaces were commonly observed within channels exposed to pressure gradients, leading to variations in separation performance during both IEF and SDS-PAGE. Bulk gel properties can also be affected by hydrodynamic flow during 
photopolymerization. In channels where bulk flow was observed, the resulting gels appeared opaque and tended to adsorb proteins during SDS-PAGE separations, resulting in reduced separation resolution. Even in channels with optically clear gel, it is likely that differing degrees of bulk flow result in variations in sieving performance. Thus new approaches to eliminating bulk flow during photopolymerization are needed to further improve separation reproducibility. It should also be noted that the use of chip-based differential gel electrophoresis (DIGE) can ultimately reduce the impact of chip-to-chip variations by enabling differential analysis of multiple samples within a single 2-D PAGE separation.

\section{Concluding remarks}

The presented 2-D microfluidic system combines multifunctional gels, a discontinuous buffer system, and novel chip design elements to achieve high-resolution multidimensional separations of E. coli cell lysate proteins. The multifunctional PAAm gels offer several important benefits for the microfluidic platform. Following previous demonstrations of PAAm gels as pressure-blocking elements in complex microfluidic systems, short plugs of in situ photopolymerized PAAm gel integrated into selected regions of the chip eliminate unwanted hydrodynamic flow, enabling the various solutions required for both IEF and SDS-PAGE to be isolated until needed during each stage of the separation process. The presented system extends the use of PAAm gel plugs as reagent containers, allowing discrete packets of SDS and discontinuous buffers to be placed on-chip during the fabrication process. This approach to on-chip reagent storage allows a substantial reduction in chip complexity by eliminating the need for separate SDS injection channels as used in our previous work, ${ }^{10}$ while also simplifying the system-level operational requirements by integrating all solutions except the sample/ampholyte mixture into the chip. Beyond the present application, gel plugs may also find use as on-chip reagent containers in other microfluidic systems requiring precise metering of reagents.

The high resolving capacity of slab gel SDS-PAGE is largely due to the use of isotachophoretic stacking of the sample prior to its introduction into the sieving gel. While the traditional Laemmli buffer system cannot be directly applied to the microfluidic platform due to the lack of a stacking gel within the chip, the novel discontinuous buffer system enabled by the on-chip gel plugs provides substantial sharpening of protein bands during SDS-PAGE. The choice of anion and cation species, ion concentration, and buffer $\mathrm{pH}$ within each portion of the system was made on the basis of extensive experiments performed over a wide range of conditions. The in-gel band sharpening is believed to result from a transient electric field or $\mathrm{pH}$ gradient generated during the injection of the highly mobile glycinate ions initially maintained at basic $\mathrm{pH}$ within the injection gel plugs. Further modeling and optimization of the buffer conditions is needed to fully explore the sharpening effect and yield additional improvements in the peak capacity of the 2-D system.

Because reagents are packaged on-chip using the multifunctional gels, the polymer chips are single-use, and long-term storage of fabricated chips prior to use is of practical concern. Stability of the on-chip reagent packets is maintained solely by slow diffusion within the fine-pore gel matrix. Separations performed within 1 hour of chip fabrication have shown no significant variations in separation performance, but longer diffusion times are likely to lead to irreproducible results. Improved longevity may be possible by freezing chips after fabrication. While more rigorous characterization is ongoing to evaluate freezing as a viable method for long-term storage of chips containing PAAm gel plugs, no significant variations in separation results have been observed for single-channel chips exposed to a $12 \mathrm{~h}$ freeze/thaw cycle at $-20{ }^{\circ} \mathrm{C}$.

\section{Acknowledgements}

This research was supported by NIH grant R01 GM072512.

\section{References}

1 J. C. Giddings, Unified Separation Science, John Wiley \& Sons, New York, 1991.

2 J. M. Hille, A. L. Freed and H. Watzig, Electrophoresis, 2001, 22, 4035-4052.

3 M. P. Washburn, D. Wolters and J. R. Yates, Nat. Biotechnol., 2001, 19, 242-247.

4 R. Westermeier and T. Naven, Proteomics in Practice, Wiley-VCH Verlag-GmbH, Weinheim, 2002.

5 X. X. Chen, H. K. Wu, C. D. Mao and G. M. Whitesides, Anal. Chem., 2002, 74, 1772-1778.

6 Y. Li, J. S. Buch, F. Rosenberger, D. L. DeVoe and C. S. Lee, Anal. Chem., 2004, 76, 742-748.

7 A. Griebel, S. Rund, F. Schonfeld, W. Dorner, R. Konrad and S. Hardt, Lab Chip, 2004, 4, 18-23.

8 C. Das, C. K. Fredrickson, Z. Xia and Z. H. Fan, Sens. Actuators A.Phys., 2007, 134, 271-277.

9 C. Das, J. Zhang, N. D. Denslow and Z. H. Fan, Lab Chip, 2007, 7, 1806-1812.

10 J. K. Liu, S. Yang, C. S. Lee and D. L. DeVoe, Electrophoresis, 2008, 29, 2241-2250.

11 C. A. Emrich, I. L. Medintz, W. K. Chu and R. A. Mathies, Anal. Chem., 2007, 79, 7360-7366.

12 S. Yang, J. K. Liu and D. L. DeVoe, Lab Chip, 2008, 8, 1145-1152.

13 R. A. Zangmeister and M. J. Tarlov, Langmuir, 2003, 19, 6901-6904.

14 M. M. Bradford, Anal. Biochem., 1976, 72, 248-254.

15 A. Guttman, J. Horvath and N. Cooke, Anal. Chem., 1993, 65, 199203

16 A. T. Andrews, Electrophoresis, Clarendon Press, Oxford, 1986.

17 A. S. Cohen and B. L. Karger, J. Chromatogr., 1987, 397, 409-417.

18 B. L. Karger and A. S. Cohen, US Patent No. 4,865,707, 1989.

19 J. J. Lu, S. R. Liu and Q. S. Pu, J. Proteome. Res, 2005, 4, 1012-1016.

20 A. E. Herr and A. K. Singh, Anal. Chem., 2004, 76, 4727-4733.

21 A. E. Herr, D. J. Throckmorton, A. A. Davenport and A. K. Singh, Anal. Chem, 2005, 77, 585-590.

22 B. J. Kirby, A. R. Wheeler, R. N. Zare, J. A. Fruetel and T. J. Shepodd, Lab Chip, 2003, 3, 5-10.

23 H. F. Yin, J. A. Lux and G. Schomburg, J., High Resolut. Chromatogr., 1990, 13, 624-627.

24 S. Hjerten and M. D. Zhu, J. Chromatogr., 1985, 346, 265-270.

25 L. Gao and S. R. Liu, Anal. Chem., 2004, 76, 7179-7186.

26 O. Smithies, Arch. Biochem. Biophys., 1962, 125-131.

27 D. L. Holmes and N. C. Stellwagen, Electrophoresis, 1991, 12, 612619.

28 J. A. Reynolds and C. Tanford, Proc. Natl. Acad. Sci. USA, 1970, 66, 1002-1007.

29 U. K. Laemmli, Nature, 1970, 227, 680-685.

30 L. Ornstein, Ann. N. Y. Acad. Sci., 1964, 121, 321-349.

31 B. J. Davis, Ann. N.Y. Acad. Sci., 1964, 121, 404- 427.

32 S. Ahuja, Handbook of Bioseparations, Academic Press, San Deigo, 2000.

33 H. C. Cui, K. Horiuchi, P. Dutta and C. F. Ivory, Anal. Chem., 2005, 77, 7878-7886. 